\title{
EVALUACIÓN DEL CONTENIDO GÁSTRICO CON ULTRASONIDO EN NIÑOS CON APENDICITIS AGUDA. ¿TIENEN ESTÓMAGO LLENO? RESULTADOS PRELIMINARES
}

\author{
Bustos Karina ${ }^{1}$, Barrios Rodrigo ${ }^{1}$, Leiva Carolina ${ }^{1}$, Escobar Daniel ${ }^{1}$, Merino Waldo ${ }^{1}$, Lara Vicente ${ }^{2}$ \\ Universidad de La Frontera. \\ 2 Hospital Hernán Henríquez Aravena.
}

Introducción: La aspiración del contenido gástrico es una seria complicación perioperatoria. El ultrasonido puede ser usado para diferenciar un estómago vacío de uno lleno y su uso puede ser de utilidad para evaluar el riesgo de aspiración en pacientes sometidos a cirugía de urgencia.

Objetivo General: Describir la evaluación del contenido gástrico con ultrasonido en pacientes pediátricos diagnosticados con apendicitis aguda para determinar el riesgo de aspiración en el perioperatorio.

Material y Métodos: Se seleccionó pacientes pediátricos con diagnóstico de apendicitis aguda ASA I y II, durante el mes de Septiembre del 2016 en el Hospital Hernán Henríquez Aravena. Posteriormente a la inducción de secuencia rápida, se utilizó ecógrafos Phillips Affinity 50G para la evaluación del contenido gástrico. En posición supino o decúbito lateral derecho. Para el estudio se definió buena ventana ecográfica la visualización de la arteria o vena mesentérica superior, aorta o VCS, y ángulo hepático izquierdo. Los dos diámetros antrales se midieron desde serosa a serosa. Para pacientes $<40$ kg se utilizó transductor lineal L12-4 y para $>40 \mathrm{~kg}$ el curvo C6-2. La fórmula utilizada para medir volumen fue $\mathrm{Vol}=-7,8+(3,5 \times \mathrm{CSA})+(0,127) \mathrm{x}$ edad (meses). Entre otros se registró: horas de ayuno e inicio del dolor abdominal.

Resultados: En el estudio se incluyeron un total de 19 pacientes, con un promedio de edad 92 meses (36-144), 36,8\% sexo femenino, IMC promedio $20 \mathrm{~kg} / \mathrm{mt}^{2}(17,9-26,45)$ y un $31,6 \%$ presentaba ayuno igual o menor a 8 horas. En la descripción ecográfica un $5,2 \%$ de los pacientes presentó mala ventana. En aquellos que presentaron una buena ventana: El 55,7\% se observó un estómago vacío, en el 38,8\% contenido sólido y $5,5 \%$ contenido líquido. El $42 \%$ de los pacientes presentó alto riesgo de aspiración y de estos 7 $(87,5 \%)$ presentan $>$ de 8 horas de ayuno.

Conclusiones: La ultrasonografía es una técnica que ofrece información para la toma de decisiones de manera rápida y fácil en el perioperatorio. En pacientes con apendicitis aguda y un ayuno superior a 8 horas, los resultados ecográficos apoyan la necesidad de administrar una inducción anestésica de secuencia rápida.

Tabla 1

\begin{tabular}{|c|c|c|c|}
\hline \multicolumn{4}{|l|}{ Ecografía } \\
\hline \multirow[t]{2}{*}{ Ventana } & Buena & Mala & \\
\hline & $18(94,8 \%)$ & $1(5,2 \%)$ & \\
\hline \multirow[t]{2}{*}{ Tipo de Contenido } & Vacío & Líquidos claros & Sólido \\
\hline & $10(55,7 \%)$ & $1(5,5 \%)$ & $7(38,8 \%)$ \\
\hline \multirow[t]{2}{*}{ Riesgo de aspiración } & Alto & & Bajo \\
\hline & $8(42 \%)$ & & $10(58 \%)$ \\
\hline \multirow[t]{2}{*}{ Riesgo alto de aspiración } & Ayuno $>8$ horas & & Ayuno $<8$ horas \\
\hline & $7(87,5 \%)$ & & $1(12,5 \%)$ \\
\hline
\end{tabular}

